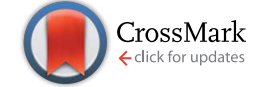

Cite this: RSC Adv., 2017, 7, 2044
Received 31st October 2016 Accepted 13th December 2016

DOI: 10.1039/c6ra26075f

www.rsc.org/advances

\title{
Preparation of a reactive flame retardant and its finishing on cotton fabrics based on click chemistry
}

\begin{abstract}
Lijin $X u^{a}$ Wei Wang ${ }^{a}$ and Dan $Y u^{* a b}$
In this study, the non-halogenated organophosphorus flame retardant dimethyl-[1,3,5-(3,5triacryloylhexahydro)triazinyl]-3-oxopropylphosphonate (DHTP) was synthesized and immobilized on cotton fabrics for a flame retardant finishing using click chemistry. The reaction and surface morphology were characterized by scanning electronic microscopy (SEM), energy dispersive spectrometer (EDS), and Fourier transform infrared spectroscopy (FTIR). Excellent flame retardancy was obtained by the limited oxygen index (LOI) and vertical burning tests. Thermal, mechanical properties, and whiteness of the treated cotton fabric were also assessed. The results demonstrate that this treatment could impart cotton fabric with flame retardancy due to the chemical bond formed between the flame retardant agent and the substrate.
\end{abstract}

\section{Introduction}

Cotton is one of the excellent natural materials that has been widely used in many fields ${ }^{\mathbf{1 , 2}}$ especially in textile industry such as carpets, curtains, and clothing. Cotton fabrics possess good hydrophilicity, softness, and hygroscopicity. Since it is highly flammable, ${ }^{3}$ the flame-retardant cotton textiles have received significant attention from both industry and academia. ${ }^{4-6}$

Traditional flame-retardant cotton textiles are mainly prepared by the flame-retardant agents containing halogens and phosphorus; however, the burning of the halogen flameretardant produces a large amount of poisonous gas, which causes secondary pollution, whereas phosphorus flame-retardant has a poor surface treatment on the textile substrates due to the lack of appropriate anchor groups for the fixation on the fiber surface. $^{7}$ Recently, DNA and proteins were used as green flameretardants; however, toxic and non-environmentally-friendly chemicals were still required for the treatment process on the textiles. ${ }^{8,9}$ In this context, the synthesis of flame retardants containing nitrogen and phosphorus with $\mathrm{N}-\mathrm{P}$ synergistic effects and their environmentally-friendly immobilization on the textiles is desirable. ${ }^{6,10}$

General methods for the flame retardant finishing on cotton involves simple impregnation or impregnation of the flame retardant, microcapsule flame-retardant technology, ${ }^{11}$ nano flame-retardant method, ${ }^{12,13}$ combination of different flame retardant systems $\mathrm{s}^{14-16}$, and application of the reactive flameretardants on the substrate. ${ }^{17-20}$ It is well known that

${ }^{a}$ College of Chemistry, Chemical Engineering and Biotechnology, Donghua University, 2999 North Renmin Road, Songjiang District, Shanghai 201620, China. E-mail: yudan@dhu.edu.cn; Fax: +86-21-67792608; Tel: +86-21-67792456

${ }^{b}$ Saintyear Holding Group Co., Ltd, China impregnation or impregnation of the flame retardant is easily operated; however, it lacks non-durable effects. Although microcapsule flame-retardant technology can reduce the migration of the liquid flame-retardant in the material and the release of toxic components during the process, there are various difficulties in choosing and designing the core and shell materials. Moreover, the control of sustained release is also troublesome. ${ }^{21}$ Some nanotechnological approaches include sol-gel, ${ }^{22,23}$ dual cure processes, ${ }^{24}$ and layer-by-layer assembly. ${ }^{22,25}$ Sol-gel derived hybrid architectures could protect the polymer surface, exerting a thermal shielding effect and thus improving the flame retardancy of the treated fabrics; however, these have some disadvantages such as difficulty in handling and discoloration. Dual cure processes require high temperatures, which damages the mechanical properties, whereas layer-by-layer assembly requires many steps during the finishing. Some methods currently used for preparing the polymer-layered silicate (PLS) nanocomposites are hard to utilize on a large scale. ${ }^{26}$ Studies demonstrated that the reactive flame retardants containing Pyrovatex CP and $N$-hydroxy-methyl compounds possess excellent durability; however, they release toxic formaldehyde. ${ }^{27,28}$ Therefore, finding an efficient flameretardant finishing method is of great interest and covalent bonding between the fibers and retardants could be a preferential technique. Click chemistry is a good synthetic tool used in drugs, polymers, functional materials, surface modification, and many other areas because of its high efficiency, versatility, and simplicity. It is also widely used in the textile functional finishes, such as antibacterial, antistatic, and flame retardant finishing, and has very good application prospects. ${ }^{29-31}$

Herein, we have applied the thiol-ene click chemistry ${ }^{32,33}$ for the flame retardant finishing of the cotton fabric. Dimethyl[1,3,5-(3,5-triacryloylhexahydro)triazinyl]-3- 


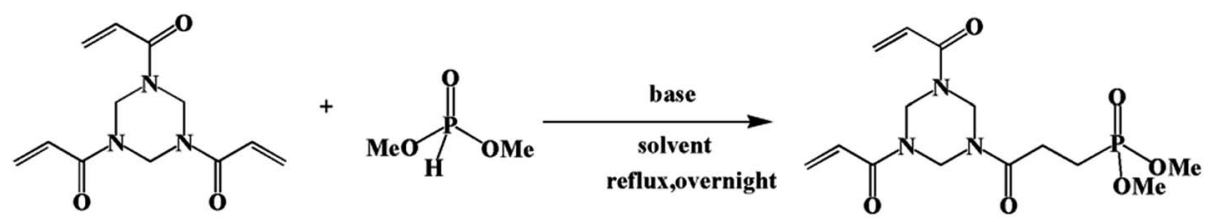

(1)

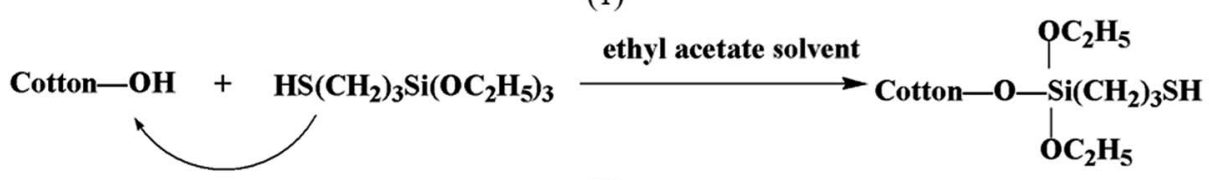

(2)

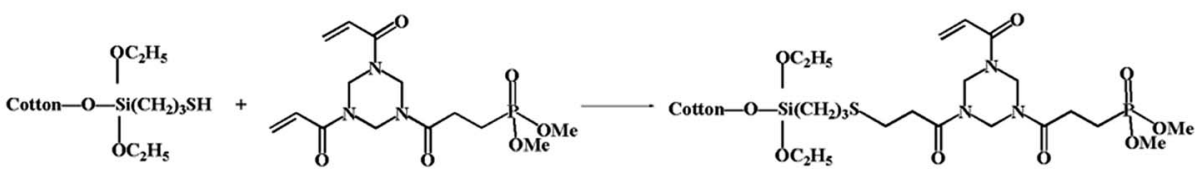

(3)

Scheme 1 Reaction eqn (1) synthesis of DHTP, (2) pretreatment of 3-mercaptopropyltriethoxysilane (MPTES), and (3) the reaction between DHTP and thiol groups by thiol-ene click chemistry.

oxopropylphosphonate (DHTP) was first synthesized according to the method reported in a study, which utilizes alkenyl as the reaction precursor. ${ }^{34}$ The thiol-reactive groups of the cotton fabric were produced through the condensation reaction between 3-mercaptopropyltriethoxysilane (MPTES) and the fiber in the presence of a catalyst. Grafting is performed by the reaction between the oxyethyl group on one end of the MPTES and hydroxyl group of the fiber surface. ${ }^{35}$ Then, the pretreated cotton fabric and DHTP with alkenyl form a chemical bond based on the thiol-ene click reaction. The related reaction equation is presented in Scheme 1. The flame retardant properties of the treated cotton fabric are mainly evaluated by the vertical flame, limiting the oxygen index, and thermogravimetric analysis.

\section{Experimental}

\subsection{Materials}

A plain cotton weave fabric of $230 \mathrm{~g} \mathrm{~m}^{-2}$ (Saintyear Holding Group Co., Ltd, China) was used in this study. 1,3,5Triacryloylhexahydro-1,3,5-triazine (TAHT), dimethyl phosphite (DP), 1,8-diazabicyclo[5.4.0]undec-7-ene (DBU), and 3-mercaptopropyltriethoxysilane (MPTES) were purchased from SigmaAldrich (USA). DHTP was synthesized using THAT and DP. ${ }^{34}$ Tris(2-carbonxyethyl)phosphine hydrochloride (TCEP) was purchased from Adamas Reagent (Switzerland). Other chemicals were analytical grade reagents and used without further purification (Sinopharm, China).

\subsection{Treatments}

THAT and DP were refluxed in chloroform for overnight in the presence of DBU. ${ }^{34}$ Pretreatment of the cotton fabric was carried out by dissolving $15 \mathrm{~g} \mathrm{~L}^{-1}$ sodium hydroxide and $7 \mathrm{~g} \mathrm{~L}^{-1}$ levelling agent $\mathrm{O}$ (alkylphenol ethoxylates) in water at $70{ }^{\circ} \mathrm{C}$ for $20 \mathrm{~min}$. Then, 3-mercaptopropyltriethoxysilane (MPTES) was dissolved in ethyl acetate and a concentration of $5 \%$ was achieved. Cotton fabrics were dipped in the MPTES solutions at room temperature for $2 \mathrm{~h}$ at a liquor ratio of $50: 1$ with mild shaking. In the subsequent process, cotton fabric was immersed in the DHTP solution with $1 \mathrm{wt} \%$ TCEP as the catalyst for $8 \mathrm{~h}$ at room temperature, and the liquor ratio was still $50: 1$ under gentle stirring. Finally, the cotton samples were rinsed with deionized water and dried at $60{ }^{\circ} \mathrm{C}$ in air. The mass was determined by weighing. The value of add-on was calculated as follows:

$$
\text { Add-on }(\%)=\frac{W_{2}-W_{1}}{W_{1}} \times 100 \%
$$

where $W_{1}$ and $W_{2}$ are the weights of the cotton fabric before and after processing, respectively.

\subsection{Characterization}

2.3.1 Scanning electronic microscopy. The untreated and treated fabric samples were sputtered gold coatings analyzed by a desktop scanning electron microscope TM-1000 (Hitachi, Japan). The SEM images were obtained in vacuum at a 3000 magnification and at accelerating voltage of $10 \mathrm{kV}$.

2.3.2 Fourier transform infrared spectroscopy. The surface functional groups were investigated by Fourier transform infrared spectroscopy (Nicolet 6700, Thermo Fisher, America). An ATR-FTIR was performed using diamond and crystal Ge for ten scans with a resolution of $4 \mathrm{~cm}^{-1}$.

2.3.3 Energy dispersive spectrometer. The chemical composition of the fiber surface was illustrated by energy dispersive spectroscopy (EDS, IE 3000, U.K.).

2.3.4 Thermogravimetric analysis. Samples were cut into pieces that weighed about $5 \mathrm{mg}$. Thermogravimetric data was analyzed by 204 F1 analyzer (Netzsch, Germany), and the samples were heated from $30{ }^{\circ} \mathrm{C}$ to $600{ }^{\circ} \mathrm{C}$ at a heating rate $10{ }^{\circ} \mathrm{C}$ $\min ^{-1}$ with a gas flow of $\mathrm{N}_{2}$. 
2.3.5 Vertical flame test. Vertical flame test was carried out according to GB/T 5455-1997 standard method using YG(B) fabric flame-retardant tester. The sample size was $30 \mathrm{~cm} \times$ $8 \mathrm{~cm}$. The ignition nozzle angle was $25^{\circ}$ and the ignition nozzle diameter was $11 \mathrm{~mm}$. Inside dimensions were $W 329 \times D 329 \times$ $H 767 \mathrm{~mm}$ and the gas type for combustion was butane. Flame height was about $40 \mathrm{~mm}$ and the burning time was $12 \mathrm{~s}$.

2.3.6 Limiting oxygen index (LOI). Limiting oxygen index (LOI) was measured according to the ASTMD2863-08 standard method using ATS1004050 oxygen index tester. Fabrics were prepared in $5 \mathrm{~cm} \times 15 \mathrm{~cm}$ and a mixture of oxygen and nitrogen was passed through a cylinder containing the fabric specimen.

2.3.7 Strength test. The strength of the treated and untreated fabric samples was tested by electronic fabric strength tester HD026N (Nantong Hongda, China). The area of sample was $25 \mathrm{~cm} \times 5 \mathrm{~cm}$ and manually tightened. Before tests, samples were placed in a constant temperature and humidity room until a constant weight was achieved, and the test was carried out in a climate chamber at a specific temperature and relative humidity.

2.3.8 Whiteness test. The whiteness index value of the treated and untreated cotton fabrics was tested by Datacolor 650 Color Measurement Spectrophotometer.

\section{Results and discussion}

\subsection{Morphology}

SEM was performed to investigate the morphology of the untreated and treated fabrics. It was found that the surface of the original fabric was smooth and exhibited a natural structure
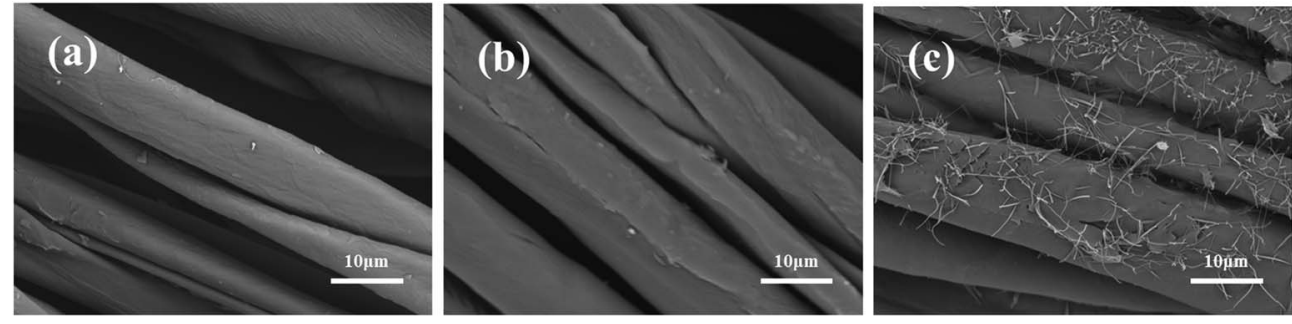

Fig. 1 SEM images of the original cotton fabric (a), MPTES treated cotton fabric (b), and the flame-retardant cotton fabric (c).

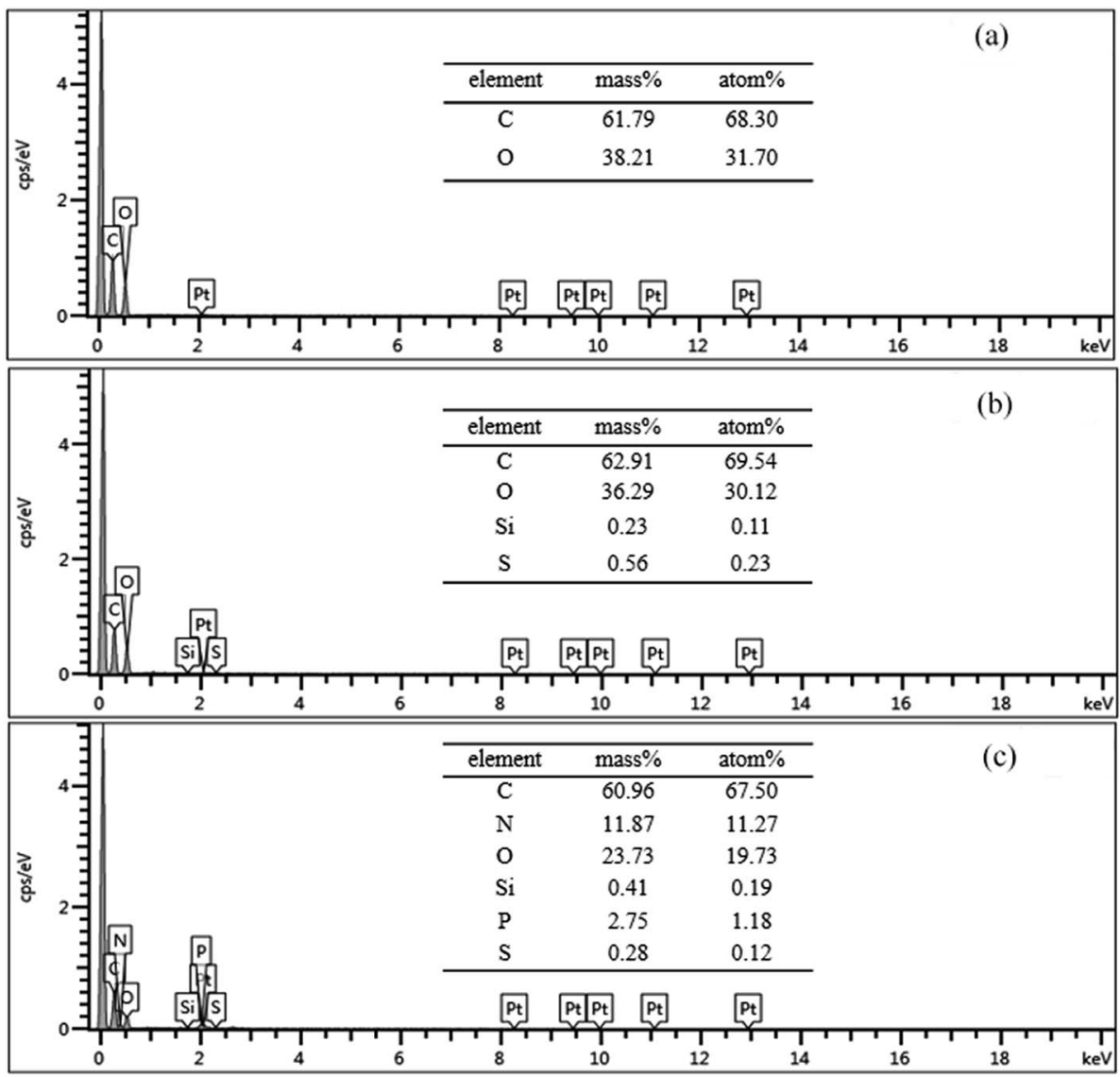

Fig. 2 EDS images of the original cotton sample (a), MPTES treated cotton sample (b), and the flame-retardant cotton sample (c). 
Table 1 EDS data for the original cotton sample (a), MPTES treated cotton sample (b), and the flame-retardant cotton sample (c)

\begin{tabular}{|c|c|c|c|c|c|c|}
\hline \multirow[b]{2}{*}{ Element } & \multicolumn{3}{|c|}{ Mass\% } & \multicolumn{3}{|c|}{ Atom \% } \\
\hline & $\mathrm{a}$ & b & $\mathrm{c}$ & a & $\mathrm{b}$ & $\mathrm{c}$ \\
\hline $\mathrm{C}$ & 61.79 & 62.91 & 60.96 & 68.30 & 69.54 & 67.50 \\
\hline $\mathrm{O}$ & 38.21 & 36.29 & 23.73 & 31.70 & 30.12 & 19.73 \\
\hline $\mathrm{Si}$ & 0 & 0.23 & 0.41 & 0 & 0.11 & 0.19 \\
\hline $\mathrm{S}$ & 0 & 0.56 & 0.28 & 0 & 0.23 & 0.12 \\
\hline $\mathrm{N}$ & 0 & 0 & 11.87 & 0 & 0 & 11.27 \\
\hline $\mathrm{P}$ & 0 & 0 & 2.75 & 0 & 0 & 1.18 \\
\hline
\end{tabular}

of grain, ravines, and distortion, as shown in Fig. 1(a). The surface of the pretreated cotton fabric became fuzzy and was cross-linked into one layer, as shown in Fig. 3(b). After further treatment with DHTP, acicular substances appeared on the surface of the cotton fibers, which may be due to the attachment of the flame retardant (Fig. 1(c)).

The chemical composition on the fiber surface was illustrated by EDS, as presented in Fig. 2 and Table 1. Comparing (a) and (b), Si and $S$ were both detected in case of (b), which were from 3-mercaptopropyltriethoxysilane. The low content of $\mathrm{Si}$ and $\mathrm{S}$ may due to the relatively low reaction ratio of the modification agents to the cotton fibers. Moreover, the flameretardant cotton sample had $\mathrm{Si}$ and $\mathrm{S}$ due to the successful pretreatment. For the flame-retardant cotton fiber (c), N and $\mathrm{P}$ peaks were observed, which were from the flame retardant DHTP, and their mass content reached $11.27 \%$ and $1.18 \%$, respectively, which indirectly confirms the success of the thiolene reaction (Fig. 2).

\subsection{Chemical reactions on the substrate surfaces}

To investigate the reaction between DHTP and the pretreated cotton fibers, the treated cotton sample was analyzed by ATRinfrared spectroscopy. The infrared spectra obtained for DHTP (a) and treated cotton fabric with DHTP (b) are shown in Fig. 3. In the spectra obtained for (a) and (b), there is a wellresolved peak near $1648 \mathrm{~cm}^{-1}$, which could be associated with

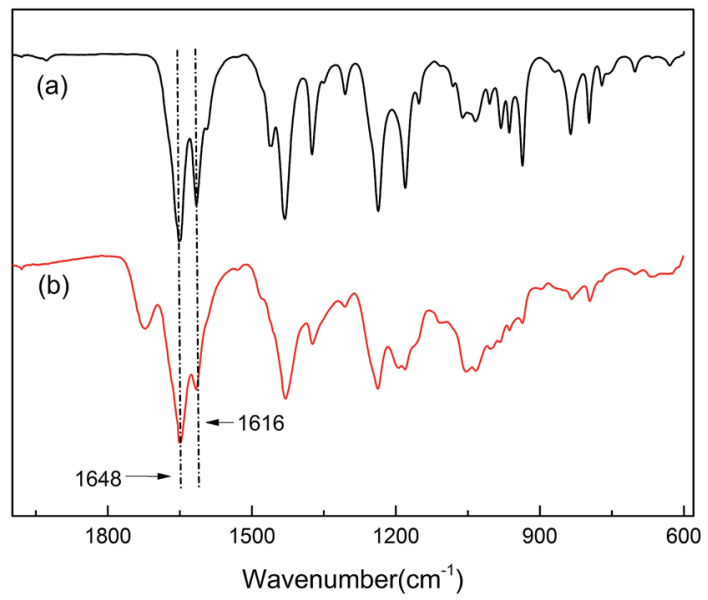

the carbonyl stretching vibration of the amide groups of the DHTP molecules. In addition, the broad band near $1616 \mathrm{~cm}^{-1}$, which was consistent with the $\mathrm{C}=\mathrm{C}$ stretch, was significantly changed in (a) and (b). ${ }^{34}$ Infrared absorption spectra of the carbonyl stretching vibration did not change for (a) and (b). However, the reduction in the intensity in features at $1616 \mathrm{~cm}^{-1}$ was clear after the treatment. This suggested that the number of $\mathrm{C}=\mathrm{C}$ bonds decreased and the reaction occurred between $\mathrm{C}=\mathrm{C}$ and thiol groups on the cotton fibers.

\subsection{Flame retardant performance}

To analyze the flame retardant performance, the original and flame-retardant cotton fabrics were subjected to LOI and vertical flame tests. Table 1 shows that the LOI value of the original cotton fabric, which was easy to burn in air, was $18.3 \%$, whereas the LOI value of the treated fabric clearly improved to 26.3\% with the effect of the flame-retardant agent DHTP. Generally, an increased LOI value results in lower combustibility and better flame-retardant property, which has been proved via the vertical flame test (Table 2). It was found that for the untreated cotton with an add-on of $28.1 \%$, after removing the ignition source, the flame quickly spread and burned the entire fabric with no residue remaining. However, for the treated cotton, after removing the ignition source, the flame immediately extinguished, and the char length was only about $5 \mathrm{~cm}$. Moreover, the treated cotton fabric was washed in water for 5 cycles, as required according to the ISO 3758-2012 standard, and the limit oxygen index test was performed. Compared to the original cotton, the LOI value was improved from $18.3 \%$ to $22.4 \%$ after the treatment.

\subsection{Thermal stability in air}

The thermal degradation process of the untreated, pretreated, and treated cotton fabrics were investigated by thermogravimetric analysis in air, as shown in Fig. 4 and Table 2 . The onset points of temperatures for the fabrics were $30.2,29.3,29.2{ }^{\circ} \mathrm{C}$,

Table 2 Vertical flame and limiting oxygen index tests

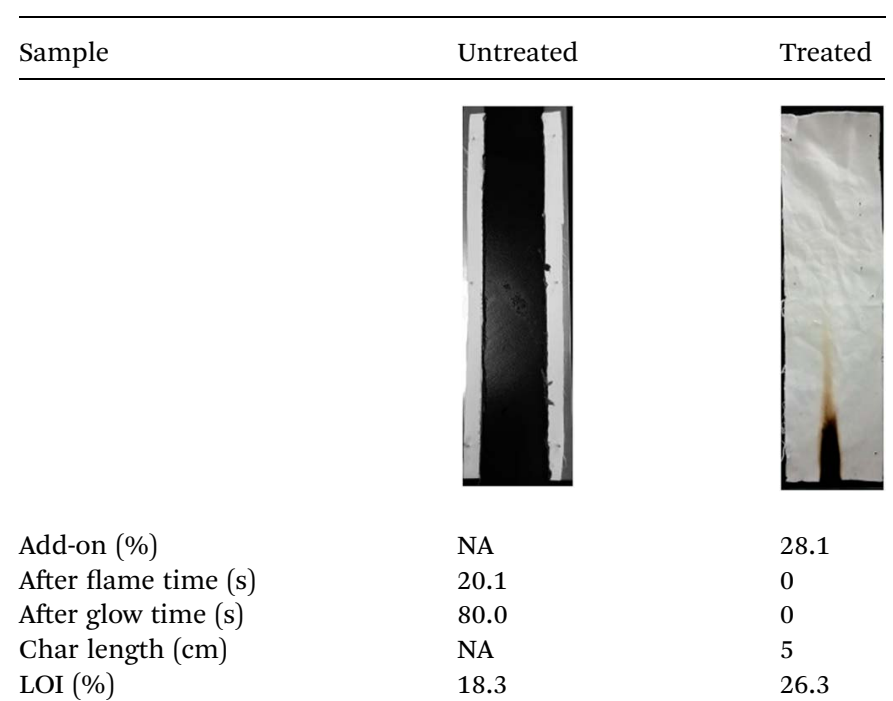

Fig. 3 IR spectra of DHTP (a) and the flame-retardant cotton fabric (b). 


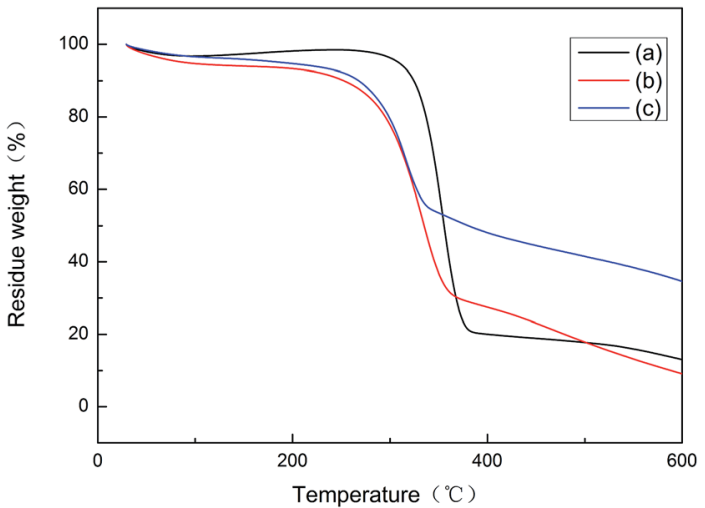

Fig. 4 TGA curves for the original cotton fabric (a), MPTES treated cotton fabric (b), and the flame-retardant cotton fabric (c).

Table 3 TGA data of the untreated and treated cotton fabrics

\begin{tabular}{|c|c|c|c|c|c|}
\hline \multirow[b]{2}{*}{ Fabrics } & \multirow[b]{2}{*}{$\begin{array}{l}T_{\text {onset }} \\
\left({ }^{\circ} \mathrm{C}\right)\end{array}$} & \multirow[b]{2}{*}{$\begin{array}{l}T_{\max }^{a} \\
\left({ }^{\circ} \mathrm{C}\right)\end{array}$} & \multirow[b]{2}{*}{$\begin{array}{l}V_{\max }{ }^{a} \\
(\% \text { per } \min )\end{array}$} & \multicolumn{2}{|c|}{ Residue at } \\
\hline & & & & $\begin{array}{l}T_{\max } \\
(\%)\end{array}$ & $\begin{array}{l}600{ }^{\circ} \mathrm{C} \\
(\%)\end{array}$ \\
\hline Untreated & 285 & 353 & -1.9 & 53.5 & 13.0 \\
\hline Pretreated & 229 & 332 & -1.0 & 52.8 & 9.1 \\
\hline Treated & 253 & 315 & -0.8 & 56.9 & 34.6 \\
\hline
\end{tabular}

${ }^{a}$ From the derivative of the TGA curves.

respectively. Weight loss of all the fabrics below $100{ }^{\circ} \mathrm{C}$ was caused by the evaporation of the adsorbed water from the cotton fabrics, and the thermal degradation stage was reached at about $300-400{ }^{\circ} \mathrm{C}$. The highest weight loss occurred as the aliphatic char and the volatiles were formed in two subsequent competitive processes, namely dehydration and depolymerisation, yielding aliphatic char and volatile products. During the weight loss stage at $400-600{ }^{\circ} \mathrm{C}$, aliphatic char is converted into aromatic structures, with the evolution of water, methane, carbon monoxide, and carbon dioxide. ${ }^{6,25}$

As observed from the results presented in Table 3, curve (b) falls slightly faster than curve (a) because MPTES reaches the decomposition temperature and the thermal stability decreases. $T_{\text {onset }}$ of the original fabric reached at $285{ }^{\circ} \mathrm{C}$, whereas $T_{\text {onset }}$ of the treated fabric was obviously reduced due to the cellulose dehydration catalyzed by DHTP in the heating process. The samples rapidly degraded between $300{ }^{\circ} \mathrm{C}$ and $400{ }^{\circ} \mathrm{C}$. The rate of weight loss for the original fabric reached

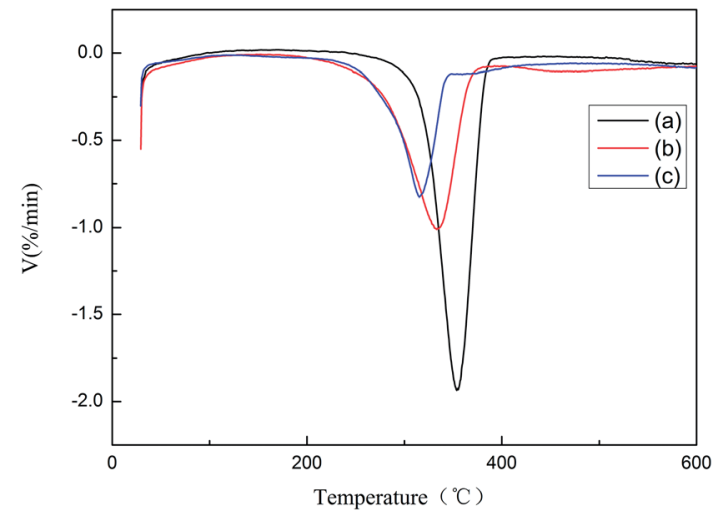

Fig. 5 DTG curves for the original cotton fabric (a), MPTES treated cotton fabric (b), and the flame-retardant cotton fabric (c).

a maximum at $353{ }^{\circ} \mathrm{C}$, and the residue at $T_{\max }$ was $53.5 \%$. However, the residue at $T_{\max }\left(315^{\circ} \mathrm{C}\right)$ of the treated fabric was $56.9 \%$, which was higher than that of the untreated sample. Moreover, the maximum decomposition rate of the flameretardant cotton was the lowest. Thus, the treated fabric had a better flame-retardant effect because of the minimal generation of the flammable gas and low decomposition rate. Furthermore, residue at $600{ }^{\circ} \mathrm{C}$ increased under the action of DHTP as well, favoring the dehydration and enhancing the stability. Moreover, $V_{\max }$ of the flame-retardant cotton fabric became slower because the phosphonic acid derivatives might have accelerated the degradation of cellulose (Fig. 5).

\subsection{Breaking strength and whiteness test}

To evaluate the mechanical properties of the treated cotton fabric, the breaking strength of the different cotton fabrics was tested according to the international standard ISO 13934-1 1999 and the results are shown in Table 4. The breaking strength-MD (warp direction) of the untreated cotton fabric is $990.3 \mathrm{~N}$. An obvious loss in the breaking strength was observed after the MPTES pretreatment because the stability weakened in the amorphous region of the cotton fibers. However, the flameretardant cotton fabric exhibited better breaking strength that increased to $1099.9 \mathrm{~N}$, which demonstrates an improvement in the mechanical properties after the DHTP treatment. The breaking strength-CD along the weft direction followed the same trend. We attributed this to the crosslinking effect of the flame-retardant agent DHTP with the thiol groups on the cotton fiber.

Table 4 Strength data of the untreated and treated cotton fabrics

\begin{tabular}{lllll}
\hline Samples & $\begin{array}{l}\text { Breaking strength } \\
\text { MD }(\mathrm{N})\end{array}$ & $\begin{array}{l}\text { Standard deviation } \\
\text { MD }(\mathrm{N})\end{array}$ & $\begin{array}{l}\text { Breaking strength } \\
\text { CD }(\mathrm{N})\end{array}$ & $\begin{array}{l}\text { Standard deviation } \\
\text { CD }(\mathrm{N})\end{array}$ \\
\hline Untreated cotton & 990.3 & 1.21 & 405.4 & 0.95 \\
MPTES treated cotton & 908.6 & 0.81 & 399.9 & 0.90 \\
Flame retardant cotton & 1099.9 & 0.92 & 473.6 & 0.68
\end{tabular}


Table 5 Whiteness data of the untreated and treated cotton fabrics

\begin{tabular}{llllllll}
\hline & \multicolumn{7}{c}{ CIE coordinates } \\
\cline { 3 - 7 } Samples & Whiteness & $L^{*}$ & $a^{*}$ & $b^{*}$ & $C^{*}$ & $H^{*}$ \\
\hline Untreated cotton & 51.27 & 91.42 & 0.67 & 6.01 & 6.05 & 83.68 \\
MPTES treated cotton & 54.65 & 91.66 & 0.62 & 5.95 & 5.99 & 83.66 \\
Flame retardant cotton & 50.46 & 92.26 & -0.19 & 6.62 & 6.62 & 84.56
\end{tabular}

The data presented in Table 5 enables to observe that how the whiteness index values changed after every cotton fabric treatments according to the international standard ISO 105-J02 1997. From the data obtained for $L^{*}$, which means the absolute lightness or darkness on a scale of black to white, the treated cotton was slightly lighter than the original cotton. The $a^{*}$ value indicates that the color of the untreated and MPTES treated cotton was reddish, whereas the flame retardant cotton was greenish. Moreover, the $b^{*}$ value showed that the three samples were yellowish. From the results obtained for $C^{*}$ and $H^{*}$, the flame retardant cotton had a deeper yellowness. Finally, the whiteness was obtained. As it was expected, the increase in the whiteness index value occurred after the MPTES treatment. However, the whiteness of the flame-retardant cotton fabric decreased. During the whole process, only a slight loss of the whiteness was observed after the flame retardant finishing, which shows that this method avoids the obvious yellowing of the fabric compared to the traditional flame retardant finishing.

\section{Conclusions}

The results of this study have demonstrated that the application of MPTES and DHTP on a cotton substrate was an effective process for achieving the flame-retardant performance of the fabric by click chemistry. From the SEM images, the changes that occurred on the cotton surface could be observed. The EDS and FT-IR analysis indicated that the reaction occurred between the ene and thiol groups on the cotton fibers. The flame retardant performance of the treated fabrics was assessed by the vertical flame test and found to be significantly better with an add-on of $28.1 \%$. The limiting oxygen index increased from $18.3 \%$ to $26.3 \%$. Compared to the original cotton, LOI of the treated cotton fabric after washing was improved from $18.3 \%$ to $22.4 \%$. The burning test results were supported by TGA. Char weight of the flameretardant cotton fabric at $600{ }^{\circ} \mathrm{C}$ was double to that of its original cotton fabric. Moreover, the treated fabric possessed excellent mechanical properties, and the whiteness of the untreated and treated fabrics was similar. All of the abovementioned results suggest that this flame retardant will be a good candidate for the flame retardant finishing of the textiles.

\section{Acknowledgements}

The research was supported by the National Natural Science Foundation of China (no. 51403032).

\section{References}

1 A. Hou and S. Gang, Carbohydr. Polym., 2013, 95, 768-772.

2 A. Hou, C. Zhang and Y. Wang, Carbohydr. Polym., 2012, 87, 284-288.

3 A. R. Horrocks, Rev. Prog. Color. Relat. Top., 1986, 16, 62-101.

4 J. Alongi, Z. Han and S. Bourbigot, Prog. Polym. Sci., 2015, 51, 28-73.

5 S. Liang, N. M. Neisius and S. Gaan, Prog. Org. Coat., 2013, 76, 1642-1665.

6 S. Wang, X. Sui, Y. Li, J. Li, H. Xu, Y. Zhong, L. Zhang and Z. Mao, Polym. Degrad. Stab., 2016, 128, 22-28.

7 M. J. Chen, Z. B. Shao, X. L. Wang, L. Chen and Y. Z. Wang, Ind. Eng. Chem. Res., 2012, 51, 9769-9776.

8 J. Alongi, R. A. Carletto, F. Bosco, F. Carosio, A. D. Blasio, F. Cuttica, V. Antonucci, M. Giordano and G. Malucelli, Polym. Degrad. Stab., 2013, 99, 111-117.

9 G. Malucelli, F. Bosco, J. Alongi, F. Carosio, A. D. Blasio, C. Mollea, F. Cuttica and A. Casale, ChemInform, 2015, 46, 46024-46039.

10 M. Neisius, T. Stelzig, S. Liang and S. Gaan, Flame retardant finishes for textiles, 2015.

11 L. Hui, Dyeing Finish., 2010, 36, 9-11.

12 E. P. Giannelis, Adv. Mater., 1996, 8, 1-9.

13 D. Q. Chen, Y. Z. Wang, X. P. Hu, D. Y. Wang, M. H. Qu and B. Yang, Polym. Degrad. Stab., 2005, 88, 349-356.

14 K. Xie, A. Gao and Y. Zhang, Carbohydr. Polym., 2013, 98, 706-710.

15 C. Q. Yang, W. Wu and Y. Xu, Fire Mater., 2005, 29, 109-120.

16 J. Alongi, M. Ciobanu and G. Malucelli, Carbohydr. Polym., 2011, 85, 599-608.

17 V. Ameri Dehabadi, H.-J. Buschmann and J. S. Gutmann, Fire Mater., 2014, 38, 166-173.

18 F. A. Abdel-Mohdy, J. Appl. Polym. Sci., 2003, 89, 2573-2578.

19 J. Ning and R. Qiu, J. Fire Sci., 1986, 4, 355-362.

20 X. Y. Liu and J. Z. Shao, J. Zhejiang Univ. Sci. Technol., 2008, 25, 261-265.

21 Y. Zhao, X. Huang, Y. Tai and D. He, Int. J. Chem., 2010, 2, 173-178.

22 G. Malucelli, F. Carosio, J. Alongi, A. Fina, A. Frache and G. Camino, Mater. Sci. Eng., R, 2014, 84, 1-20.

23 J. Alongi and G. Malucelli, J. Mater. Chem., 2012, 22, 2180521809.

24 J. Alongi, M. Ciobanu and G. Malucelli, Cellulose, 2011, 18, 1335-1348.

$25 \mathrm{~J}$. Alongi and G. Malucelli, RSC Adv., 2015, 5, 24239-24263.

26 E. P. Giannelis, Adv. Mater., 1996, 8, 1-9.

27 J. P. Guan, G. Q. Chen, G. U. Dong hua and L. I. Qiang, Dyeing Finish., 2004, 21, 11-15.

28 M. E. Hall, A. R. Horrocks and H. Seddon, Polym. Degrad. Stab., 1999, 64, 505-510.

29 D. Yu, J. Y. Cai, J. S. Church and L. Wang, ACS Appl. Mater. Interfaces, 2014, 6, 1236-1242.

30 J. Guo, Z. Xie, R. T. Tran, D. Xie, D. Jin, X. Bai and J. Yang, Adv. Mater., 2014, 26, 1906-1911. 
31 J. E. Moses and A. D. Moorhouse, Chem. Soc. Rev., 2007, 38, 1249-1262.

32 X. M. He, G. T. Zhu, Y. Y. Zhu, X. Chen, Z. Zhang, S. T. Wang, B. F. Yuan and Y. Q. Feng, ACS Appl. Mater. Interfaces, 2014, 6, 17857-17864.

33 O. D. Mcnair, B. J. Sparks, A. P. Janisse, D. P. Brent, D. L. Patton and D. A. Savin, Macromolecules, 2013, 46, 5614-5621.
34 M. Yoshiokatarver, B. D. Condon, M. S. Cintrón, S. C. Chang, M. W. Easson, C. A. Fortier, C. A. Madison, J. M. Bland and T. M. D. Nguyen, Ind. Eng. Chem. Res., 2012, 51, 1103111037.

35 D. Sun, W. Wang and D. Yu, Mater. Lett., 2016, 185, 514-518.

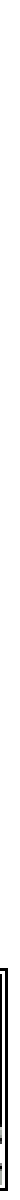

列

